\title{
Publisher Correction: Core microbiomes for sustainable agroecosystems
}

Hirokazu Toju, Kabir G. Peay (D), Masato Yamamichi (D), Kazuhiko Narisawa, Kei Hiruma, Ken Naito, Shinji Fukuda, Masayuki Ushio, Shinji Nakaoka, Yusuke Onoda, Kentaro Yoshida (1), Klaus Schlaeppi, Yang Bai, Ryo Sugiura, Yasunori Ichihashi, Kiwamu Minamisawa and E. Toby Kiers

Correction to: Nature Plants https://doi.org/10.1038/s41477-018-0139-4, published online 30 April 2018.

Owing to a technical error, this Perspective was originally published without its received and accepted dates; the dates "Received: 31 December 2017; Accepted: 23 March 2018” have now been included in all versions. 